\title{
POLYNOMIAL IDENTITY ALGEBRAS WITH INVOLUTION
}

\section{SUSAN MONTGOMERY}

\author{
ABSTRACT. It has been shown by W. Baxter and W. S. Martin- \\ dale that if $R$ is an algebra with involution over a field $F$ of char- \\ acteristic not 2, and the symmetric elements of $R$ are algebraic and \\ satisfy a polynomial identity, then $R$ is locally finite. This paper \\ extends their result to an arbitrary field, giving a new proof which \\ is independent of the characteristic of $F$.
}

There are a number of results in the literature considering problems of the following sort: Given a ring with an involution such that the symmetric elements of the ring satisfy a certain property, under what conditions is the entire ring forced to satisfy the same property? One of the most striking such results is the theorem finally proved in its full generality by S. A. Amitsur [1]: If the symmetric elements of a ring with involution satisfy a polynomial identity, then the entire ring satisfies a polynomial identity. The result with which we are most concerned is a theorem of W. Baxter and W. S. Martindale [2] stating that if $R$ is an algebra over a field $F$ such that $\frac{1}{2} \in F$, and the symmetric elements of $R$ are algebraic and satisfy a polynomial identity, then $R$ is locally finite. The purpose of this paper is to extend Baxter and Martindale's theorem to an arbitrary field $F$, giving a proof which is independent of the characteristic of $F$.

It should be pointed out that it is impossible simply to generalize Baxter and Martindale's proof. For, when $\frac{1}{2} \in F$, every element of $R$ may be written as a sum of a symmetric and a skew element, and thus every element is a sum of algebraic elements. By a theorem of Procesi [4], $R$ will then be locally finite if $R$ satisfies a polynomial identity. Since this method will not work when $F$ has characteristic 2 , completely different methods are needed.

Notice that this result generalizes a theorem of Kaplansky stating that an algebraic algebra satisfying a polynomial identity is locally finite [3, p. 167].

We will denote the involution on $R$ by ${ }^{*}$; so * is an antiautomorphism of period 2. The symmetric elements of $R$, denoted by $S$, consist of all $r \in R$ such that $r^{*}=r . R$ satisfies a polynomial identity

Received by the editors May 13, 1970.

AMS 1968 subject classifications. Primary 1649, 1658.

Key words and phrases. Rings with involution, polynomial identity, algebraic algebras.

Copyright (1) 1971, American Mathematical Society 
(P.I.) of degree $d$ over $F$ if there is a nonzero element $f\left(t_{1}, \cdots, t_{n}\right)$ of degree $d$ in the free algebra over $F$ generated by the $t_{i}$ such that $\left(r_{1}, \cdots, r_{n}\right)=0$, for all $r_{1}, \cdots, r_{n} \in R$.

The following lemma is perhaps well known, but does not seem to appear in the literature.

Lemma. If $R$ is a ring with a right quotient ring $Q$ and $R$ has an involution *, then * can be extended to an involution of $Q$.

Proof. ${ }^{1}$ First, * can be considered, in particular, as an isomorphism *: $R \rightarrow R^{0}$ where $R^{0}$ is the ring anti-isomorphic to $R$. Therefore, $R$ and $R^{0}$ have both left and right quotient rings; it is clear, in fact, that $Q^{0}$ is the left and right quotient ring of $R^{0}$. Now by the universal property of quotient rings, the diagram

$$
\begin{aligned}
& R \stackrel{*}{\rightarrow} R^{0} \\
& \downarrow \downarrow \downarrow \\
& Q \quad Q^{0}
\end{aligned}
$$

can be completed uniquely to a map $Q^{*} \rightarrow Q^{0}$. This is an isomorphism and $*^{2}: Q \rightarrow Q$ is an automorphism extending $1: R \rightarrow R$. Therefore, again by universality, $*^{2}=1$ on $Q$, and the lemma is proved.

We are now in a position to prove the main

THEOREM. If $R$ is a P.I. algebra over any field $F, R$ has an involution * and $S$ is algebraic over $F$, then $R$ is algebraic over $F$. In addition, $R$ is locally finite.

Proof. We first show that without loss of generality, we may assume $R$ is a prime ring. For, if $R$ is not algebraic, there is some $x \in R$ which is not algebraic. Let

$$
A=\{p(x), \text { nonzero polynomials in } x \text { with coefficients in } F\} .
$$

Note that $0 \notin A$ since $x$ is not algebraic, and that $A$ is multiplicatively closed.

Let $I$ be an ideal maximal with respect to excluding $A$; it is easy to see that $I$ is a prime ideal. Thus, $R / I=\bar{R}$ is a prime ring. Now $\bar{x}$, the image of $x$, is not algebraic in $\bar{R}$ because of the definition of $I$. Consider the case when $I^{*} \Phi I$. Then $I+I^{*} \Phi I$, so $\left(I+I^{*}\right) \cap A \neq 0$ by the maximality of $I$. Let $p(x) \in\left(I+I^{*}\right) \cap A$. Since $p(x) \in I+I^{*}, p(x)$ $=a+b$, where $a \in I, b \in I^{*}$. Now $b \in I^{*}$ implies that $b^{*} \in I$, and so $b+b^{*}=p(x)+\left(b^{*}-a\right) \equiv p(x)(\bmod I)$. Since $b+b^{*}$ is algebraic, $p(\bar{x})$ is

1 The proof of this lemma is the referee's. The author's own proof was much longer. 
algebraic in $\bar{R}$. But then $\bar{x}$ is algebraic, a contradiction. Thus, it must happen that $I^{*}=I$.

In this case, $\bar{R}$ has an induced involution (if $\bar{a}$ is the image of $a \in R$, as $\bar{a}^{*}$ is given by $\left.\overline{a^{*}}\right) . \bar{R}$ satisfies the same P.I. as $R$, as it is a homomorphic image of $R$, and, in addition, every symmetric element of $\bar{R}$ is algebraic. For, say $\bar{a}=\bar{a}^{*}$. Then

$$
\bar{a}^{2}=\bar{a} \bar{a}^{*}=\overline{a a^{*}},
$$

so $\vec{a}^{2}$ is the image of a symmetric element of $R$, and so is algebraic. But then $\bar{a}$ itself is algebraic. Summarizing, $\bar{R}$ is a prime P.I. ring with ${ }^{*}$ such that the symmetric elements are algebraic. If $\bar{R}$ were algebraic, we would have a contradiction, since $\bar{x}$ is not algebraic. Thus, we may assume that $R$ is prime.

By a theorem of Posner [3, p. 184], a prime ring satisfying a P.I. has a right quotient ring $D_{m}$, the $m \times m$ matrices over a division ring $D$, where $D$ is finite dimensional over its center $Z$. Thus, to show that $R$ is algebraic over $F$, it will be enough to show that $Z$ is algebraic over $F$.

As a first step, extend the involution on $R$ to an involution on $D_{m}$; this is possible by the lemma. Then to show that $Z$ is algebraic, it will suffice to show that the symmetric elements of $Z$ are algebraic. For, given $z \in Z, z+z^{*}$ and $z^{*} z$ are symmetric. If these elements are algebraic, so is $z$, since $z$ satisfies the polynomial $x^{2}-\left(z+z^{*}\right) x+z^{*} z=0$.

Let $z$ be any element of $Z$ with $z^{*}=z$. We may write $z=a b^{-1}$, some $a, b \in R$, so $z b b^{*}=a b^{*}$. Since $z \in Z,\left(a b^{*}\right)^{*}=\left(z b b^{*}\right)^{*}=b b^{*} z=z b b^{*}=a b^{*}$. That is, $a b^{*}$ is symmetric. But then $a b^{*}$ is algebraic; also $\left(b b^{*}\right)^{-1}$ is algebraic, since $b b^{*}$ is symmetric and thus algebraic. This means that $z=\left(a b^{*}\right)\left(b b^{*}\right)^{-1}$, a product of algebraic elements of $D_{m}$. Since $z \in Z$, $a b^{*}$ and $\left(b b^{*}\right)^{-1}$ commute, and thus $z$ itself is algebraic.

To see that $R$ is also locally finite, apply the theorem of Kaplansky $[3$, p. 167] stated in the introduction.

CoROLlary 1. If $R$ is an algebra with *over $F$ such that $S$ is algebraic and satisfies $a$ P.I., then $R$ is algebraic, P.I., and locally finite.

Proof. By the theorem of Amitsur mentioned previously [1], $R$ satisfies a P.I. since $S$ does. We may now use the theorem proved above.

CoRollary 2. If $R$ is an algebra with * over $F$ such that $S$ is algebraic of bounded degree, then $R$ is algebraic, P.I., and locally finite.

Proof. If $S$ is algebraic of bounded degree, then $S$ satisfies a P.I. $[3$, p. 155], so the result follows from Corollary 1 . 


\section{REFERENCES}

1. S. A. Amitsur, Rings with involution, Israel J. Math. 6 (1968), 99-106. MR 39 \#256.

2. W. E. Baxter and W. S. Martindale III, Rings with involution and polynomial identities, Canad. J. Math. 20 (1968), 465-473. MR 36 \#5168.

3. I. N. Herstein, Non-commutative rings, Carus Math. Monographs, no. 15, Math. Assoc. of America; distributed by Wiley, New York, 1968. MR $37 \# 2790$.

4. C. Procesi, The Burnside problem, J. Algebra 4 (1966), 421-425. MR 35 \#2956.

DePaul University, Chicago, Illinois 60614 